\title{
Introduction to "Particle Metropolis-Hastings using gradient and Hessian information” by J. Dahlin, F. Lindsten, T. Schön
}

\author{
Christophe Andrieu
}

Published online: 6 November 2014

(C) Springer Science+Business Media New York 2014

The authors investigate the use of metropolis adjusted Langevin algorithms (MALA) in the context of particle MCMC algorithms. The ability to use this type of updates can lead to more efficient MCMC algorithms. The challenge in this context is that MALA and more sophisticated versions require the evaluation of the gradient of the loglikelihood and/or its Hessian, which are not available analytically. A way around this consists of estimating these quantities numerically. This can be achieved efficiently by exploiting the particle filter output used to compute the estimator of the likelihood function required to implement particle MCMCs. This idea was proposed originally in Doucet et al. (2011) and similar ideas have been subsequently investigated in Nemeth and Fearnhead (2014) and Dahlin et al. (2013) and Dahlin et al. (2014).

The authors' contribution here is to propose solutions to the fact that the estimated Hessian can be negative and an extensive numerical evaluation of the performance properties of the algorithm which results in some guidelines concerning the choice of the parameters of the algorithm. The main conclusion of this investigation is that the use of this type of strategy can bring significant benefits for a negligible computational overhead.

\section{References}

Dahlin, J., Lindsten, F., Schön, T.B.: Particle metropolis Hastings using Langevin dynamics. In: Proceedings of the 38th International Conference on Acoustics, Speech, and Signal Processing (ICASSP), Vancouver (2013)

Dahlin, J., Lindsten, F., Schön, T.B.: Second-order particle MCMC for Bayesian parameter inference. In: Proceedings of the 19th IFAC World Congress, Cape Town (2014)

Doucet, A., Jacob, P., Johansen, A.M.: Discussion on Riemann manifold Langevin and Hamiltonian Monte Carlo methods. J. Royal Stat. Soc. 73(2), 162 (2011)

Nemeth, C., Fearnhead, P.: Particle metropolis adjusted Langevin algorithms for state-space models. Pre-print arXiv:1402.0694v1 (2014)
C. Andrieu ( $\varangle)$

Department of Mathematics, University of Bristol,

Bristol BS8 1TW, UK

e-mail: c.andrieu@bris.ac.uk 